\title{
论 文
}

\section{基于压力驱动节点配水量模型的供水管网震后 水力分析}

\author{
韩朝 $^{1,2}$, 马东辉 ${ }^{2,3}$, 侯本伟 ${ }^{1 *}$, 王威 ${ }^{2,3}$ \\ 1. 北京工业大学建筑工程学院, 北京 100124; \\ 2. 北京工业大学抗震减灾研究所, 北京 100124 ; \\ 3. 北京工业大学建筑与城市规划学院, 北京 100124 \\ *E-mail: benweihou@bjut.edu.cn
}

收稿日期: 2017-12-08; 接受日期: 2018-05-14; 网络版发表日期: 2019-01-03

国家自然科学基金(批准号: 51508528, 51678017)和国家科技支撑计划(批准号: 2015BAK14B01)资助项目

\begin{abstract}
摘要 本文提出了一个基于压力驱动节点配水量模型的供水管网震后供水能力评价方法. 采用Monte Carlo模拟 分析得到管网震后供水满足率均值, 作为管网震后供水能力评价结果; 在单次模拟分析中采用随机抽样方法确定 管道地震破坏状态, 并形成管网震后水力模型, 基于压力驱动节点配水量模型进行管网震后水力平差计算. 在案 例分析中, 分别应用本文方法与美国Cornell大学开发的供水管网震后水力功能分析软件GIRAFFE评价某城市供 水管网在不同地震烈度作用下的供水能力. 结果表明, 本文方法分析结果与GIRAFFE分析结果的变化规律一致, 相同模拟收玫条件下本文方法所需Monte Carlo模拟运行次数少于GIRAFFE软件, 本文方法进行单次Monte Carlo 模拟的计算时间少于GIRAFFE软件; 在基于压力驱动节点配水量模型的管网震后水力分析模型中, 管道破坏造成 的漏水量会同时降低该管道上游和下游节点供水满足率, 与管网震害实际情况相符.
\end{abstract}

关键词 供水管网, 震后水力分析, Monte Carlo模拟, 压力驱动节点配水量

\section{1 引言}

供水管网系统是城市生命线工程的重要组成部 分, 是维持城市基本功能的重要基础设施. 强烈地震作 用下, 大量供水管道被破坏, 导致供水管网供水能力下 降, 进而影响灾后城市恢复和重建. 分析供水管网震后 状态是发现供水管网抗震薄弱环节, 并进行抗震设防 的基础.

供水管网震后状态分析方法包括管网震后水力分
析方法和管网震后连通性分析方法 ${ }^{[1,2]}$, 本文采用管网 震后水力分析方法，管网震后水力分析方法是在震前 管网正常运行水力模型中添加地震产生的管道破坏点 后, 形成管网震后水力模型, 并对管网震后水力模型进 行水力平差计算, 得到节点震后水压及配水量.

美国Cornell大学O'Rourke教授团队 ${ }^{[3 \sim 6]}$ 在管道地 震破坏状态模型、破坏管道漏水模型、供水管网震后 水力分析模型等问题的研究基础上, 开发了供水管网 震后水力功能分析软件(GIRAFFE). GIRAFFE采用

引用格式: 韩朝, 马东辉, 侯本伟, 等. 基于压力驱动节点配水量模型的供水管网震后水力分析. 中国科学: 技术科学, 2019, 49: 351-362 Han Z, Ma D H, Hou B W, et al. Post-earthquake hydraulic analyses of urban water supply network based on pressure drive demand model (in Chinese). Sci Sin Tech, 2019, 49: 351-362, doi: 10.1360/N092017-00429 
Monte Carlo方法确定管网震后的平均供水能力; 在单 次Monte Carlo模拟中随机生成管道破坏点位置和破 坏类型，根据节点固定需水量进行水力平差，采用隔 离负压节点的方法确定管网震后水力状态 ${ }^{[3]}$. 其中, 生 成管道破坏类型、渗漏形态、漏口面积的概率模型是 根据Northridge地震中管道破坏的统计数据建立的 ${ }^{[7]}$. Wang和 $A u^{[8]}$ 在GIRAFFE的基础上, 分析供水管网功能 可靠度的空间分布, 并识别影响管网抗震能力的关键 管道. 马东辉等人 ${ }^{[9]}$ 利用GIRAFFE软件对供水管网进 行震后水力分析. 目前, GIRAFFE软件被洛杉矶水电 部门作为地震灾害预防决策的辅助工具.

传统的供水管网水力分析中假设管网压力可以满 足用户需求, 每个用户需水量均得到满足, 地震破坏后 供水管网处于多点漏水, 低压运行状态. 部分节点需水 量不能全部满足，此时节点实际配水量是与节点压力 相关的. 若仍用固定需水量代替实际配水量进行管网 水力平差计算, 根据满足节点流量连续性方程, 为满 足节点固定需水量而降低节点水压，计算结果中会出 现不符合实际情况的节点负压. GIRAFFE软件假设供 水管网震后节点配水量仍为震前的固定需水量, 计算 结果中存在节点负压. 为了处理节点负压问题, GIRAFFE采用隔离负压节点的方法, 节点震后配水量只存 在节点震前需水量的 $100 \%$ 或 0 , 这导致其水力平差结 果不准确 ${ }^{[10,11]}$. 压力驱动节点配水量分析模型(PDD) 考虑节点配水量与节点水压关系，更适合低压状态下 管网水力分析 ${ }^{[12 ~ 14]}$. 许多学者对压力驱动节点配水量 分析模型在供水管网震后水力分析中的应用进行了探 索 ${ }^{[10,13,15 \sim 17]}$.

本文基于压力驱动节点配水量分析模型，调用 EPANET软件提出的函数工具包epanet $2 . d 11$ 进行供水 管网震后水力平差, 采用Monte Carlo方法得到供水管 网震后平均供水能力, 并将本文方法与GIRAFFE软件 方法的计算结果对比.

\section{2 压力驱动节点配水量模型}

压力驱动节点配水量模型假设：当节点水压达到 或超过服务水压 $\left(H^{\mathrm{des}}\right)$ 时，节点配水量等于节点需水 量; 当节点水压低于或等于最低水压 $\left(H^{\mathrm{min}}\right)$ 时，节点配 水量为 0 ; 当节点水压在服务水压与最低水压之间时, 节点配水量与节点水压满足一定的函数关系. 采用
Wagner等人 ${ }^{[17]}$ 提出的节点水压和配水量关系，如式(1) 所示:

$Q_{i}^{*}= \begin{cases}0, & H_{i}<H_{i}^{\text {min }}, \\ Q_{i} \cdot \sqrt{\frac{H_{i}-H_{i}^{\text {min }}}{H_{i}^{\text {des }}-H_{i}^{\text {min }}}}, & H_{i}^{\text {min }} \leq H_{i}<H_{i}^{\text {des }}, \\ Q_{i}, & H_{i}^{\text {des }} \leq H_{i},\end{cases}$

式中, $Q_{i}$ 为节点 $i$ 的需水量 $(\mathrm{L} / \mathrm{s}) ; Q_{i}{ }^{*}$ 为考虑节点水压和 配水力关系的节点 $i$ 的配水量 $(\mathrm{L} / \mathrm{s}) ; H_{i}$ 为节点 $i$ 水压 $(\mathrm{m})$; $H_{i}^{\mathrm{min}}$ 为节点 $i$ 配水量 $Q_{i}{ }^{*}$ 大于 0 所需的最低节点水压 $(\mathrm{m})$; $H_{i}^{\mathrm{des}}$ 为节点 $i$ 满足需水量 $Q_{i}$ 的服务水压 $(\mathrm{m})$.

\section{3 供水管网震后水力分析方法}

Monte Carlo模拟方法计算供水管网震后供水满 足率的均值 $\mu_{N}$ 作为最终评价结果; 单次Monte Carlo模 拟主要分为三部分: 生成地震后管道破坏、建立管网 震后水力模型以及基于压力驱动节点配水量分析模型 的水力平差计算, 分析方法过程如图1所示.

输出结果包括: 各次Monte Carlo模拟的供水管网 震后供水满足率为 $S S I_{k}, k$ 次模拟中 $i$ 节点的供水满足率 $S I_{i}{ }^{k}$, 各次模拟的供水管网震后供水满足率的均值为 $\mu_{N}$, 分别按下式计算:

$$
\begin{aligned}
& S S I_{k}=\frac{\left(\sum_{i=1}^{m} Q_{i}^{k}\right)}{\sum_{i=1}^{m} Q_{i}}, \\
& S I_{i}{ }^{k}=\frac{Q_{i}^{k}}{Q_{i}}, \\
& \mu_{N}=\frac{1}{N} \sum_{k=1}^{N} S S I_{k},
\end{aligned}
$$

式中, $Q_{i}^{k}$ 和 $Q_{i}$ 分别为第 $i$ 节点在第 $k$ 次Monte Carlo模拟 中的实际配水量和震前配水量 $(\mathrm{L} / \mathrm{s}) ; m$ 为供水管网中 用户节点数量; $N$ 为Monte Carlo模拟次数.

\section{1 生成地震后管道破坏点}

(1) 确定管道破坏点个数及位置

假设地震后管道发生破坏是随机独立事件, 且沿 管道长度 $L$ 服从泊松分布, 则管道发生震害的概率为

$P_{\mathrm{f}}=1-\exp (-R R \cdot L)$,

式中, $P_{\mathrm{f}}$ 为地震后管道发生破坏的概率; $R R$ 为管道平 


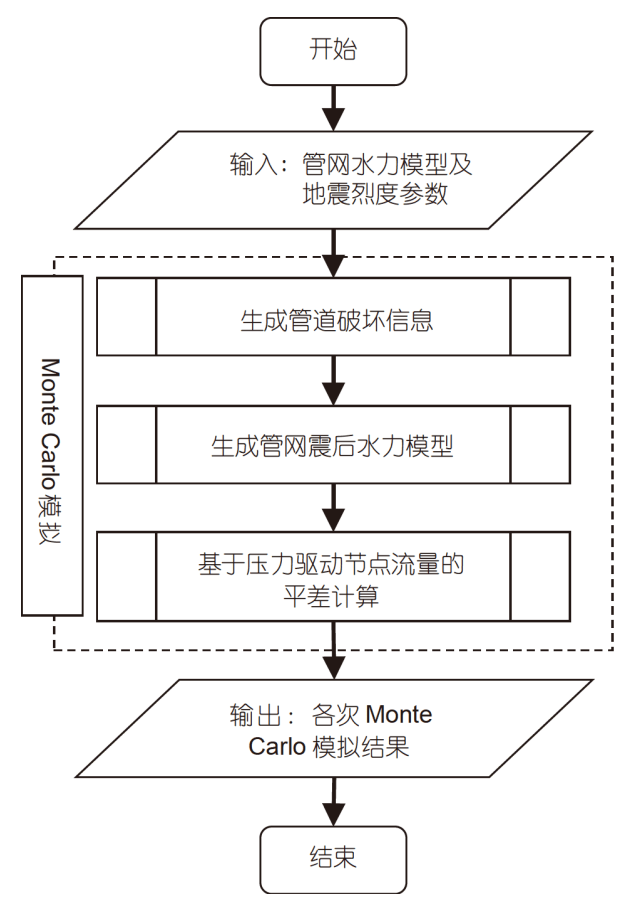

图 1 供水管网震后水力分析方法流程图

均震害率 $($ 处 $/ \mathrm{km}) ; L$ 为管道长度 $(\mathrm{km})$.

生成地震后管道破坏点位置的过程如图2所示: $L_{1}$ 为破坏点 1 与管道起点 $\mathrm{A}$ 距离, $L_{k}$ 为破坏点 $k$ 与破坏点 $k-1$ 距离, 当直到 $\sum_{1}{ }^{n} L_{k}>L$ 时, 停止生成破坏点. $L_{k}$ 为 服从均值为 $1 / R R$ 的指数分布的随机数, 按式(6)计算.

$L_{k}=\frac{1}{R R} \ln \left(1-\mu_{\mathrm{a}}\right)$,

式中, $L_{k}$ 为第 $k$ 次破坏点与第 $k-1$ 次破坏点之间管道长 度 $(\mathrm{km}) ; \mu_{\mathrm{a}}$ 为服从在 $0 \sim 1$ 之间均匀分布的随机数.

计算管道平均震害率 $R R$ 时, 采用 Jeon和 $\mathrm{O}^{\prime} \mathrm{R}-$ ourke ${ }^{[7]}$ 以及Wang 和 ${ }^{\prime}$ Rourke ${ }^{[6]}$ 对 1994年Northridge地 震的地震动数据及供水管线震害数据进行拟合得到的 计算公式.

球墨铸铁管:

$\ln (R R)=1.84 \cdot \ln (\mathrm{PGV})-9.40$;

灰口铸铁管:

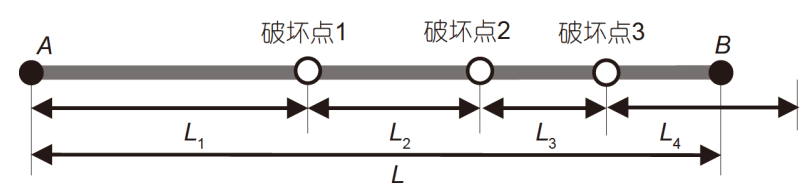

图 2 管道生成破坏点示意 $\ln (R R)=1.21 \cdot \ln (\mathrm{PGV})-6.81$

石棉水泥管:

$\ln (R R)=2.26 \cdot \ln (\mathrm{PGV})-11.10 ;$

钢管(铆接接头, $D \geq 600 \mathrm{~mm}$ ):

$\ln (R R)=1.41 \cdot \ln (\mathrm{PGV})-8.19$;

钢管(焊接接头, $D \geq 600 \mathrm{~mm}$ ):

$\ln (R R)=2.59 \cdot \ln (\mathrm{PGV})-14.16$;

钢管(焊接接头, $D<600 \mathrm{~mm}$ ):

$\ln (R R)=0.75 \cdot \ln (\mathrm{PGV})-4.80$,

式中, $P G V$ 为地面峰值速度 $(\mathrm{cm} / \mathrm{s})$.

(2) 确定管道破坏点类型

美国生命线协会 $(\mathrm{ALA})^{[18]}$ 将管道破坏分为两种: 断开和渗漏. 断开为管道从中间完全分离, 管道丧失全 部输水能力; 而渗漏为管道破口, 管道丧失部分输水能 力. 美国建筑科学协会(NIBS)的调查 ${ }^{[19]}$ 表明, 地震后管 道破坏 $80 \%$ 为渗漏破坏, $20 \%$ 为断开破坏. Monte Carlo 模拟抽样时，对每个生成的管道破坏点，在 $0 \sim 1$ 之间生 成随机数 $\mu_{\mathrm{b}}$, 若 $\mu_{\mathrm{b}}<0.2$, 则破坏点为断开破坏; 否则, 即 为渗漏破坏.

(3) 确定管道渗漏形式及渗漏开口面积

Shi和O'Rourke ${ }^{[5]}$ 依据不同管材与接头形式将管 道渗漏分为 5 种形式: 接头环向松动、横向裂缝、纵向 裂缝、管壁破损、管壁撕裂，并给出了不同形式的发 生概率及对应的渗漏开口面积计算公式，适用于各类 常用的承插式接口管道和连续式无接口管道. 表 1 显 示了 5 种渗漏形式对应不同管材的发生概率. 表 2 给出 5 种渗漏形式相应的渗漏开口面积 $A_{L}$ 估算方法. 按照 表1中渗漏形式发生概率，随机抽样确定管道渗漏破 坏的渗漏形式，按照表 2 公式计算相应的渗漏开口面 积 $A_{L}$.

\section{2 破坏点水力模型}

(1) 管道断开点水力模型

如图3(a)所示, 当长度为 $L$ 的管道 $\mathrm{AB}$ 上发生断开破 坏, 破坏点与起点 $\mathrm{A}$ 距离为 $\lambda L$. 对应水力模型如图3(b) 所示, 在管道断开位置增加两个虚拟水池, 分别与节点 $\mathrm{A}$ 和 $\mathrm{B}$ 连接, 连接的管道长度分别为 $\lambda L$ 与 $(1-\lambda) L$, 其他 如直径、管材等参数与管道 $\mathrm{AB}$ 相同. 根据 $\lambda L$ 在端点 $\mathrm{A}$ 和 $\mathrm{B}$ 的高程之间线性插值得到虚拟水池的高程. 断开 
表 1 各种管道渗漏形式的概率 ${ }^{[5]}$

\begin{tabular}{cccccc}
\hline 管材 & 环向松动 & 横向裂缝 & 纵向裂缝 & 管壁破损 & 管壁撕裂 \\
\hline 普通铸铁 & 0.3 & 0.5 & 0.1 & 0.1 & - \\
球墨铸铁 & 0.8 & - & 0.1 & 0.1 & - \\
钢管(铆接) & 0.6 & - & 0.3 & - & - \\
钢管(焊接) & - & - & - & - & - \\
混凝土管 & 1.0 & - & - & - & - \\
\hline
\end{tabular}

表 2 管道渗漏形式说明及渗漏面积计算公式(根据文献[5]整理)

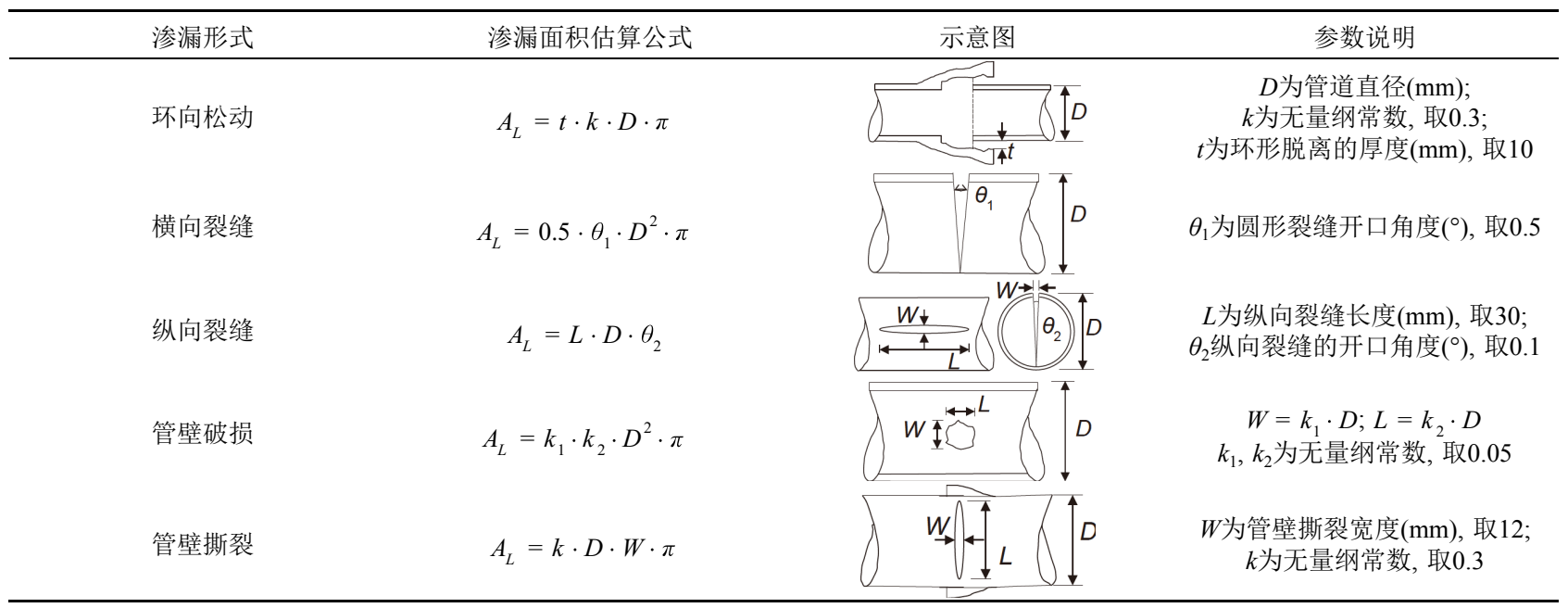

(a)

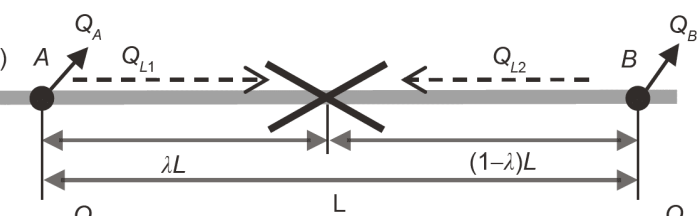

(b)

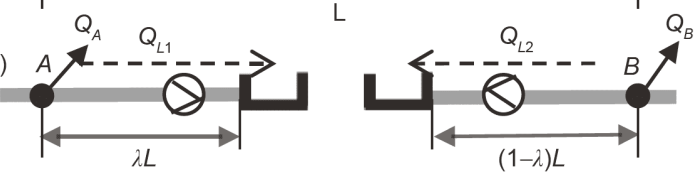

(c)

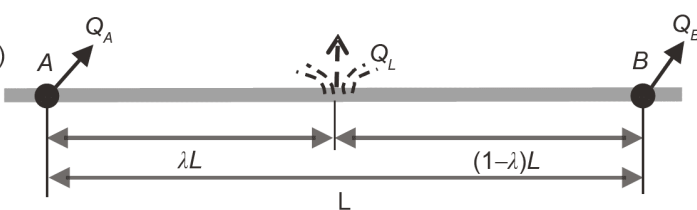

(d)

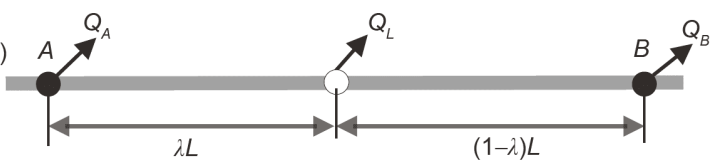

图 3 管道破坏的示意图与水力模型. (a) 管道断开示意图; (b) 管道断开水力模型; (c) 管道渗漏示意图; (d) 管道渗漏水 力模型
点处流量应满足管内压力水头与孔口处局部水头损失 关系. 管道断开处漏水量 $\left(Q_{L 1}, Q_{L 2}\right)$ 可由管道端点(图3 (b)中的 $\mathrm{A}, \mathrm{B})$ 的压力水头在断开管道长度 $(\lambda L,(1-\lambda) L)$ 上的沿程水头损失关系得到 ${ }^{[20]}$.

(2) 管道渗漏点破坏水力模型

如图3(c) 所示, 当长度为 $L$ 的管道 $\mathrm{AB}$ 上发生渗漏破 坏，破坏点与起点 $\mathrm{A}$ 距离为 $\lambda L$. 对应水力模型如图3(d) 所示, 在管道破坏位置增加一个扩散器, 分别与端点 $\mathrm{A}$ 和 $\mathrm{B}$ 连接, 连接管道长度分别为 $\lambda L$ 与 $(1-\lambda) L$, 其他如直 径、管材等参数与管道 $\mathrm{AB}$ 相同. 根据 $\lambda L$ 在端点 $\mathrm{A}$ 和 $\mathrm{B}$ 的高程之间线性插值得到扩散器的高程. 扩散器的流 量 $Q_{L}$ 可按照EPANET中扩散器模型 ${ }^{[21]}$ 计算.

$Q_{L}=C_{1} \cdot \mu \cdot A_{L} \cdot \sqrt{H}$,

式中, $Q_{L}$ 为扩散器流量 $(\mathrm{L} / \mathrm{s}), C_{1}$ 为单位转换系数, 当 $Q$ 与 $A_{L}$ 单位分别为 $\mathrm{L} / \mathrm{s}$ 和 $\mathrm{m}^{2}$ 时, 取 $4427 ; \mu$ 为孔口流量系, 取值范围为 $0.60 \sim 0.90 ; A_{L}$ 为渗漏面积 $\left(\mathrm{m}^{2}\right)$, 按表 2 中相 
应渗漏形式计算; $H$ 为破坏处水压 $(\mathrm{m})$.

\section{3 基于压力驱动节点配水量模型的平差计算}

将破坏点的水力模型添加到震前供水管网正常运 行的水力模型, 修改震前管网的拓扑结构, 形成管网震 后拓扑结果. 与GIRAFFE水力计算方法的区别在于: GARIFFE软件采用的方法中，处理节点负压时，在管 网震后拓扑结构的基础上, 需要再删除负压节点及邻 近管道; 管网震后拓扑结果是随着负压节点的位置而 发生改变的. 本文采用基于压力驱动节点配水量模型 的平差计算方法, 在管网震后拓扑结构中进行水力分 析并处理节点负压时, 只需调整节点配水量, 不再对 管网震后拓扑结果进行修改，本文方法既考虑了低压 状态下节点需水量部分满足的实际情况, 又避免了 GIRAFFE水力计算时, 处理负压节点过程中修改管网 拓扑结构, 提高运算速率.

调用EPANET toolkit中epanet2.dll函数进行管网水 力平差. 在水力平差过程中, 基于压力驱动节点配水量 模型采用迭代方法逐步调整节点配水量, 充分发挥了 epanet2.dll进行水力平差的计算性能. 核心计算过程如 图4所示, 其中 $Q_{i}^{j}$ 和 $H_{i}^{i}$ 分别为第 $j$ 次迭代得到的节点 $i$ 的 配水量和水压; $Q^{*}$ 为考虑节点水压和配水力关系的节 点的配水量 $(\mathrm{L} / \mathrm{s})$, 按式(1)计算; $\Delta$ 为两次迭代计算节点 配水量的相对误差, 按式(14)计算; $\delta$ 为计算精度; $N_{2}$ 为 最大迭代次数.

$$
\Delta=\frac{\left|Q_{i}^{j+1}-Q_{i}^{j}\right|}{Q_{i}^{j}} .
$$

\subsection{Monte Carlo模拟结束条件}

(1) Monte Carlo模拟次数 $N_{1}$

设置计数器 $k$, 当第一次执行Monte Carlo模拟时, 令 $k=1$; 此后, 每执行一次Monte Carlo模拟, 令 $k+1$; 当 $k=N_{1}$ 时, 停止Monte Carlo模拟, 并输出各次Monte Car10 模拟中管网震后水力分析结果.

(2) Monte Carlo模拟结果收敛条件

随着Monte Carlo模拟次数增加, 各次模拟的供水 管网震后供水满足率的均值 $\mu_{N}$ 变化逐渐减小. 当 $\mu_{N}$ 变 化足够小时, Monte Carlo模拟结果趋于稳定, 可以结 束Monte Carlo模拟.

选择各次Monte Carlo模拟结果 $S S I_{k}$ 的均值 $\mu_{N}$ 和变

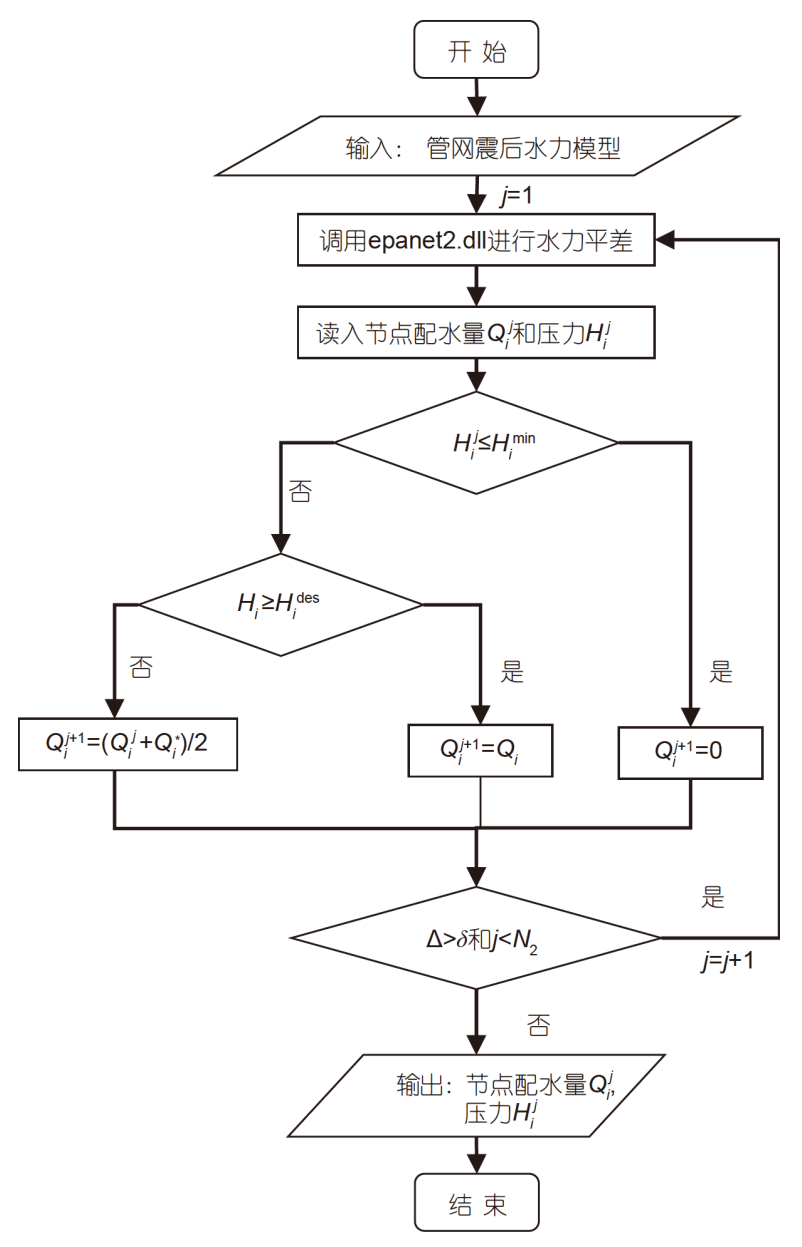

图 4 基于压力驱动节点配水量模型的平差计算流程图

异系数Cov变化率小于指定精度 $\xi$ 作为Monte Carlo模 拟结束条件. $S S I_{k}$ 的均值 $\mu_{N}$ 和变异系数 $\mathrm{Cov}$ 变化率分别 为 $\eta_{\mu}$ 和 $\eta_{c}$. 算法将先执行 10 次Monte Carlo模拟, 然后每 隔 5 次计算 $\eta_{\mu}$ 和 $\eta_{c}$, 计算公式如下:

$\eta_{\mu}=\left|\mu_{N}-\mu_{N-5}\right|$,

$\eta_{c}=\left|\frac{\sigma_{N}}{\mu_{N}}-\frac{\sigma_{N-5}}{\mu_{N-5}}\right|$,

式中, $\mu_{N}, \mu_{N-5}$ 分别为 $N$ 次和 $N-5$ 次Monte Carlo模拟的 $S S I_{k}$ 均值; $\sigma_{N}, \sigma_{N-5}$ 分别为 $N$ 次和 $N-5$ 次Monte Carlo模拟 的 $S S I_{k}$ 标准差.

\section{4 案例分析}

案例供水管网 ${ }^{[22]}$ 的拓扑结构及水源供水分区如 图5所示, 节点的高程与需水量见表 3 , 管道的长度与直 


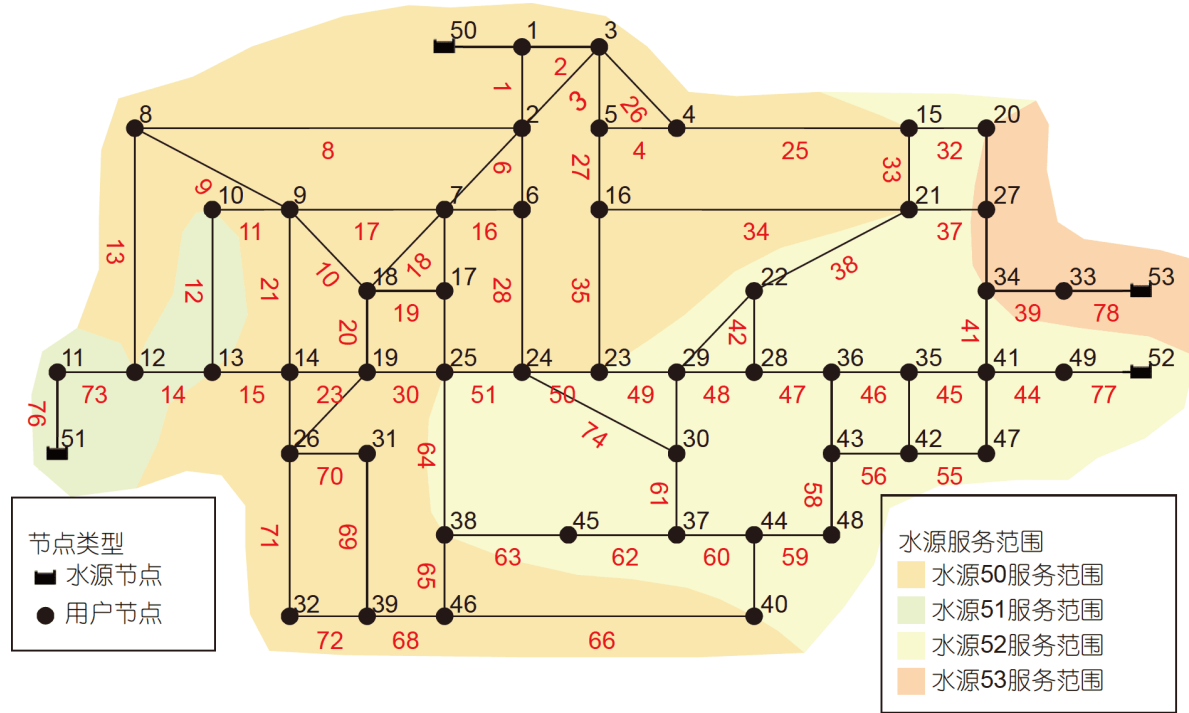

图 5 供水管网模型及供水区域

表 3 案例管网节点信息

\begin{tabular}{|c|c|c|c|c|c|c|c|c|c|c|c|c|c|c|}
\hline 编号 & $\begin{array}{c}\text { 高程 } \\
(\mathrm{m})\end{array}$ & $\begin{array}{c}\text { 需水量 } \\
(\mathrm{L} / \mathrm{s})\end{array}$ & 编号 & $\begin{array}{c}\text { 高程 } \\
(\mathrm{m})\end{array}$ & $\begin{array}{c}\text { 需水量 } \\
(\mathrm{L} / \mathrm{s})\end{array}$ & 编号 & $\begin{array}{c}\text { 高程 } \\
(\mathrm{m})\end{array}$ & $\begin{array}{c}\text { 需水量 } \\
(\mathrm{L} / \mathrm{s})\end{array}$ & 编号 & $\begin{array}{c}\text { 高程 } \\
(\mathrm{m})\end{array}$ & $\begin{array}{c}\text { 需水量 } \\
(\mathrm{L} / \mathrm{s})\end{array}$ & 编号 & $\begin{array}{c}\text { 高程 } \\
(\mathrm{m})\end{array}$ & $\begin{array}{c}\text { 需水量 } \\
(\mathrm{L} / \mathrm{s})\end{array}$ \\
\hline 1 & 9.2 & 40 & 11 & 12.9 & 27 & 21 & 9.3 & 116 & 31 & 9.2 & 14 & 41 & 30.5 & 80 \\
\hline 2 & 8 & 82 & 12 & 17.6 & 174 & 22 & 11.5 & 286 & 32 & 20.5 & 141 & 42 & 12 & 137 \\
\hline 3 & 10.2 & 56 & 13 & 12 & 147 & 23 & 18 & 194 & 33 & 9.6 & 96 & 43 & 12.1 & 72 \\
\hline 4 & 10 & 69 & 14 & 10.6 & 151 & 24 & 10 & 100 & 34 & 18 & 85 & 44 & 15 & 39 \\
\hline 5 & 11 & 99 & 15 & 9 & 47 & 25 & 10.8 & 138 & 35 & 18.8 & 82 & 45 & 9.1 & 38 \\
\hline 6 & 15 & 233 & 16 & 11.8 & 76 & 26 & 13.3 & 76 & 36 & 11.7 & 123 & 46 & 9.6 & 28 \\
\hline 7 & 12 & 121 & 17 & 14.5 & 122 & 27 & 9 & 83 & 37 & 9.4 & 86 & 47 & 15.5 & 408 \\
\hline 8 & 11.4 & 266 & 18 & 10.9 & 123 & 28 & 10.4 & 220 & 38 & 9.1 & 191 & 48 & 18.8 & 28 \\
\hline 9 & 10.5 & 276 & 19 & 10 & 120 & 29 & 11.2 & 168 & 39 & 9.8 & 47 & 49 & 9.7 & 94 \\
\hline 10 & 12 & 205 & 20 & 10.6 & 59 & 30 & 9.5 & 117 & 40 & 12 & 210 & & & \\
\hline
\end{tabular}

径见表4. 其中节点 $50,51,52,53$ 为水源点, 总水头分别 为: $76.95,74.31,83.58,81.98 \mathrm{~m}$; 管道1的Hazen-Williams粗糙系数为 80 , 管道 $44,45,46,49$ 的粗糙系数为 90 , 其余管道的粗粘系数为 120 . 假定直径大于 $600 \mathrm{~mm}$ 的管道，为钢管(铆接接头). 采用式(10)计算管道平均 震害率 $R R$; 直径小于 $600 \mathrm{~mm}$ 的管道, 管材为球墨铸铁 (橡胶密封接头), 采用式(7)计算管道平均震害率 $R R$. Monte Carlo模拟和压力驱动节点配水量模型相关参 数取值为 $\xi=0.02 ; \delta=0.01 ; N_{2}=100 ; H_{i}^{\mathrm{min}}=0 \mathrm{~m} ; H_{i}^{\mathrm{des}}=$ $10 \mathrm{~m}$. 根据《中国地震烈度表》(GB/T17742-2008)选 择地震烈度为VII, VIII, IX度时, 地面峰值速度PGV和
管道平均震害率 $R R$ 见表 5 .

\section{1 本文方法与GIRAFFE评价结果对比分析}

应用GIRAFFE软件设置模拟结束条件方法分析 管网在地震烈度为VII, VIII和IX度下供水管网供水能 力, 模拟结束条件为: $\eta_{\mu}<\xi$ 和 $\eta_{c}<\xi$, 并记录GIRAFFE软 件运行Monte Carlo模拟次数. 然后, 将本文方法与 GIRAFFE软件进行相同次数的Monte Carlo随机模拟, 结果见表6和图6.

如表6所示，本文方法评价管网SSI与GIRAFFE评 价结果有相同的趋势, 即随着地震烈度提高, 管网 SSI 
表 4 案例管网管道信息

\begin{tabular}{|c|c|c|c|c|c|c|c|c|c|c|c|c|c|c|}
\hline 编号 & $\begin{array}{c}\text { 长度 } \\
(\mathrm{m})\end{array}$ & $\begin{array}{c}\text { 直径 } \\
(\mathrm{mm})\end{array}$ & 编号 & $\begin{array}{c}\text { 长度 } \\
(\mathrm{m})\end{array}$ & $\begin{array}{l}\text { 直径 } \\
(\mathrm{mm})\end{array}$ & 编号 & $\begin{array}{c}\text { 长度 } \\
\text { (m) }\end{array}$ & $\begin{array}{l}\text { 直径 } \\
(\mathrm{mm})\end{array}$ & 编号 & $\begin{array}{c}\text { 长度 } \\
\text { (m) }\end{array}$ & $\begin{array}{l}\text { 直径 } \\
(\mathrm{mm})\end{array}$ & 编号 & $\begin{array}{c}\text { 长度 } \\
(\mathrm{m})\end{array}$ & $\begin{array}{l}\text { 直径 } \\
(\mathrm{mm})\end{array}$ \\
\hline 1 & 1200 & 1400 & 17 & 1500 & 500 & 33 & 1400 & 200 & 49 & 950 & 850 & 65 & 680 & 600 \\
\hline 2 & 1400 & 900 & 18 & 1100 & 500 & 34 & 2900 & 500 & 50 & 950 & 500 & 66 & 550 & 500 \\
\hline 3 & 800 & 300 & 19 & 900 & 500 & 35 & 2700 & 700 & 51 & 900 & 500 & 67 & 2800 & 700 \\
\hline 4 & 2000 & 300 & 20 & 1400 & 500 & 36 & 1200 & 500 & 52 & 3000 & 700 & 68 & 1000 & 500 \\
\hline 5 & 1300 & 900 & 21 & 1200 & 850 & 37 & 1050 & 500 & 53 & 3150 & 500 & 69 & 1400 & 300 \\
\hline 6 & 2100 & 750 & 22 & 1600 & 500 & 38 & 1200 & 500 & 54 & 1200 & 500 & 70 & 650 & 500 \\
\hline 7 & 2600 & 600 & 23 & 700 & 500 & 39 & 1000 & 500 & 55 & 1500 & 500 & 71 & 1400 & 500 \\
\hline 8 & 3500 & 1000 & 24 & 1000 & 600 & 40 & 1500 & 500 & 56 & 1000 & 500 & 72 & 1000 & 500 \\
\hline 9 & 800 & 1000 & 25 & 1750 & 300 & 41 & 1000 & 600 & 57 & 1100 & 500 & 73 & 900 & 500 \\
\hline 10 & 600 & 700 & 26 & 2300 & 500 & 42 & 2000 & 300 & 58 & 3600 & 1000 & 74 & 1100 & 500 \\
\hline 11 & 1300 & 300 & 27 & 1100 & 900 & 43 & 2900 & 500 & 59 & 800 & 1000 & 75 & 1742 & 1000 \\
\hline 12 & 1300 & 300 & 28 & 2600 & 500 & 44 & 3000 & 1600 & 60 & 1750 & 600 & 76 & 6636.3 & 500 \\
\hline 13 & 3700 & 500 & 29 & 750 & 600 & 45 & 1700 & 1400 & 61 & 1100 & 500 & 77 & 4262.8 & 1000 \\
\hline 14 & 1450 & 500 & 30 & 1300 & 500 & 46 & 850 & 1400 & 62 & 550 & 500 & 78 & 8577.1 & 500 \\
\hline 15 & 500 & 500 & 31 & 1600 & 500 & 47 & 1400 & 1100 & 63 & 480 & 400 & & & \\
\hline 16 & 1400 & 600 & 32 & 500 & 300 & 48 & 1100 & 1100 & 64 & 2820 & 700 & & & \\
\hline
\end{tabular}

表 $5 R R$ 计算结果

\begin{tabular}{cccc}
\hline 地震烈度 & VII & VIII & IX \\
\hline PGV $(\mathrm{cm} / \mathrm{s})$ & 13 & 25 & 50 \\
$R R(D \geq 600 \mathrm{~mm})$ & 0.0103 & 0.0260 & 0.0690 \\
$R R(D<600 \mathrm{~mm})$ & 0.0246 & 0.0542 & 0.1254 \\
\hline
\end{tabular}

表 6 模拟结果

\begin{tabular}{cccc}
\hline 地震烈度 & VII & VIII & IX \\
\hline 模拟次数 & 20 & 25 & 20 \\
$\mu$-SSI (GIRAFFE) & 0.962 & 0.891 & 0.698 \\
$\mu-S S I$ (本文方法) & 0.978 & 0.937 & 0.837 \\
\hline
\end{tabular}

均值降低; 如图6所示, 在相同地震烈度, 相同模拟次数 条件下, GIRAFFE与本文方法模拟各个节点供水满足 率SI变化趋势一致. 说明, 本文方法分析结果的变化规 律与GIRAFFE分析结果一致.

\section{2 本文方法评价结果合理性分析}

(1) 抽样结果合理性分析

在地震烈度为XI度时，分别从 GIRAFFE模拟和本 文方法Monte Carlo模拟结果序列 SSI中分别选择

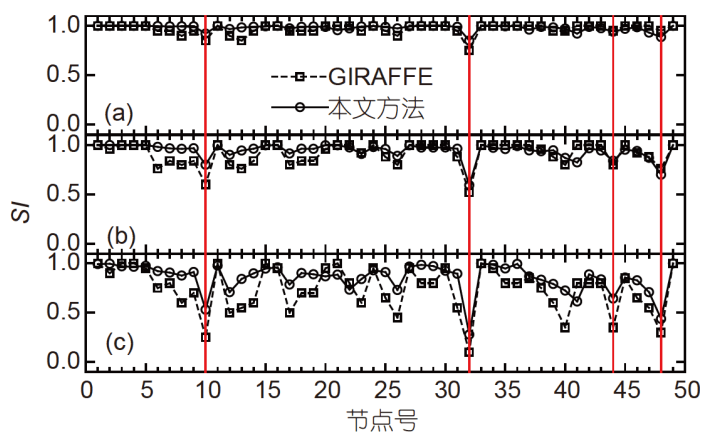

图 6 节点供水满足率分布. (a) 地震烈度为VII度; (b) 地震 烈度为VIII度; (c) 地震烈度为IX度

$S S I_{\text {median }}$ 为中位数的工况. GIRAFFE的Monte Carlo模 拟中选取管网震后水力模型 $\mathrm{a}\left(\right.$ 模型a), $S S I_{\text {median }}^{\mathrm{a}}=0.682$; 本文方法的Monte Carlo模拟中选取管网震后水力模 型 $\mathrm{b}$ (模型 $\mathrm{b}), S S I^{\mathrm{b}}{ }_{\text {median }}=0.854$, 这说明GIRAFFE模拟结 果SSI整体上比本文方法模拟结果低，管网破坏如图7 所示.

图8为各个管道破坏概率以及在模型 $\mathrm{a}$ 和模型 $\mathrm{b}$ 发 生破坏的管道. 可以看出, 模型 $\mathrm{b}$ 中发生破坏管道的破 坏概率普遍高于模型 $\mathrm{a}$. 说明模型 $\mathrm{b}$ 更符合“管道破坏概 率 $P_{\mathrm{f}}$ 高的管道更容易发生破坏”的事实. 


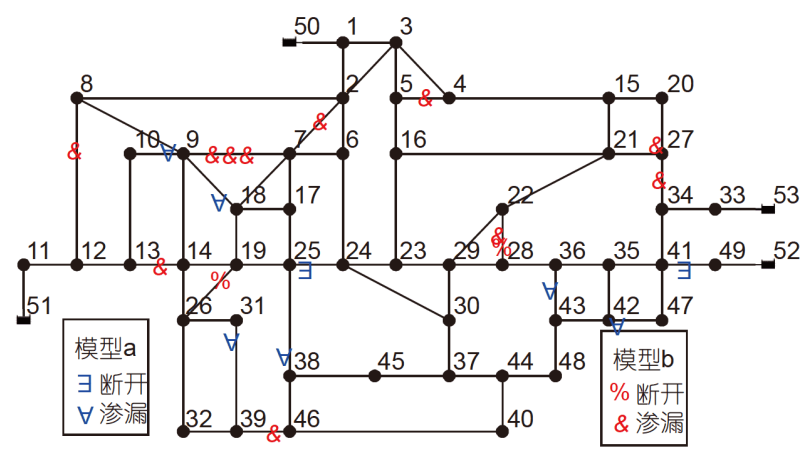

图 7 模型 $\mathrm{a}$ 和模型 $\mathrm{b}$ 的拓扑结构示意图

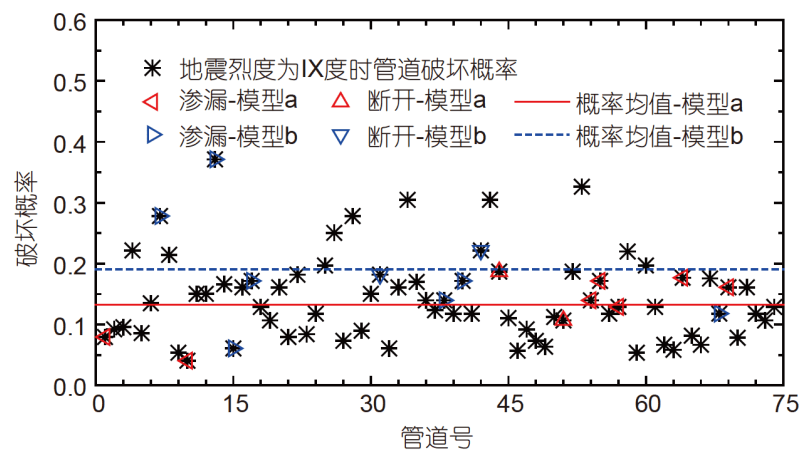

图 8 管道破坏概率及模型 $\mathrm{a}$ 和模型 $\mathrm{b}$ 中破坏管道

\section{(2) 水力平差合理性分析}

分别采用本文采用的基于压力驱动节点配水量模 型的水力平差方法(PDD方法)与GIRAFFE采用的节点 固定需水量驱动分析的水力平差方法(DDA方法)对模 型 $\mathrm{a}$ 和模型 $\mathrm{b}$ 进行水力平差, 结果如图9所示. 对于模型 $a$, 基于压力驱动节点配水量模型的水力平差方法得到 的供水管网供水满足率高于固定节点配水量的水力平 差方法; 而对于模型b, 则结果相反. 进一步分析, 这是 由于在DDA方法计算模型b时， 22 个节点(约 $45 \%$ )水压 在0 10 m之间(图10). 在PDD方法中, 水压在0 10 m之 间的节点需水量部分满足; 而在GIRAFFE计算中水压 在0 10 m之间的节点需水量完全满足，并未考虑低压 $(0 \sim 10 \mathrm{~m})$ 对节点需水量的影响. 因此, GIRAFFE评价 节点供水满足率偏高.

当管网破坏较严重时，即地震后管网中大量节点 水压 $H_{i}<0 \mathrm{~m}$ 时, PDD方法评价供水满足率高于GIRAFFE. 而当管网破坏较轻时，即地震后管网中大量节 点水压 $H_{i}$ 在0 10 m之间时, GIRAFFE得到的管网供水 满足率偏高.

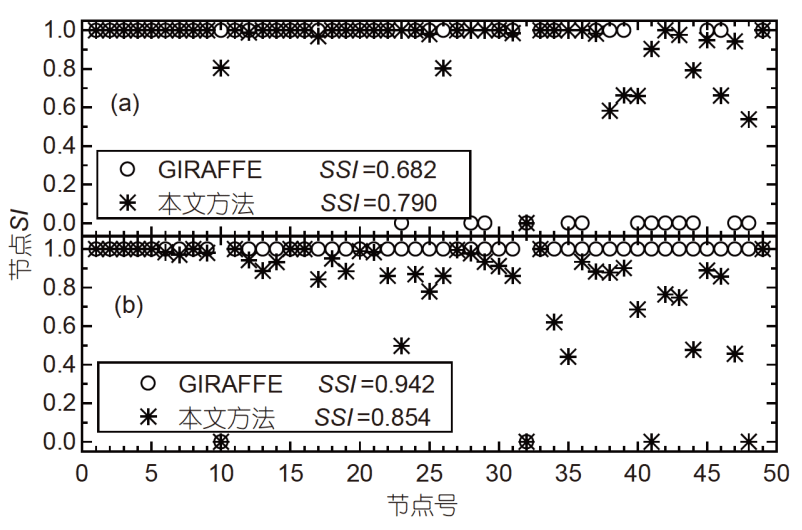

图 9 两种方法分别分析管网水力模型的供水满足率. (a) 管网震后水力模型 $\mathrm{a}$; (b) 管网震后水力模型b

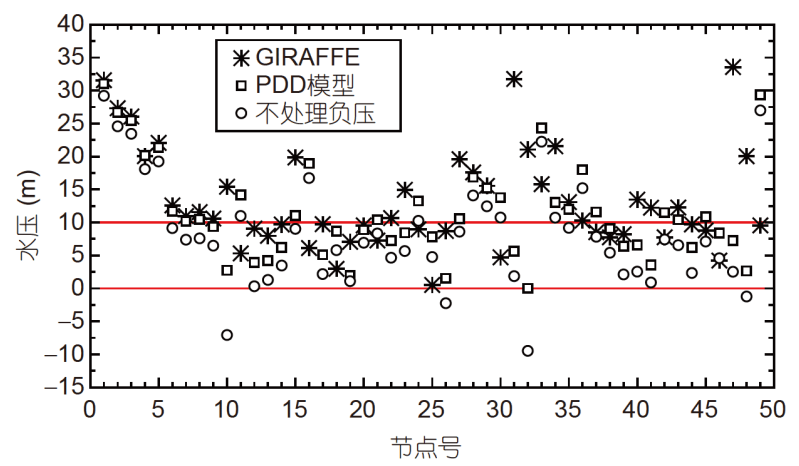

图 10 不同方法计算管网模型 $\mathrm{b}$ 的节点水压

\section{3 模拟效率分析}

(1) 相同的模拟次数下模拟时间

分别应用本文方法与GIRAFFE在地震烈度为VII, VIII, IX度下进行 1000次Monte Carlo模拟, 记录模拟所 需时间如表7所示. 可以看出, 进行 1000次Monte Carlo 模拟，本文方法所需时间少于GIRAFFE，并且烈度越 高, 本文方法所需时间 $t_{1}$ 与 GIRAFFE所需时间 $t_{2}$ 的比值 越小. 这是因为GIRAFFE在进行管网震后水力分析时, 当出现节点压力为负时，需要不断删除负压节点及邻 接管道，不断重新生成管网拓扑结构; 并且地震烈度 越高, 负压节点越多, 需要重新生成管网拓扑结构的 次数越多. 而本文方法采用PDD方法进行管网震后水 力分析, 无需重新生成管网拓扑结构, 可以充分发挥 epanet2.dl1的计算性能, 因此进行相同次数的Monte Carlo模拟本文方法所需时间更少, 且地震烈度越高, 与GIRAFE所需时间比值越小.

(2) 相同的收敛条件下模拟次数 
表 7 1000次Monte Carlo模拟所用时间(s)

\begin{tabular}{cccc}
\hline 地震烈度 & 本文方法 $\left(t_{1}\right)$ & $\operatorname{GIRAFFE}\left(t_{2}\right)$ & 比值 $\left(t_{2} / t_{1}\right)$ \\
\hline VII & 276.56 & 307.39 & 1.11 \\
VIII & 357.95 & 676.43 & 1.89 \\
IX & 458.03 & 1021.70 & 2.23 \\
\hline
\end{tabular}

在相同的收玫条件下, 即 $\xi=0.02$, 分别进行本文方 法与GIRAFFE模拟, 记录模拟结束时Monte Carlo模拟 次数, 结果如表 8 所示. 可以看出, 在相同收玫条件下, 地震烈度为VII，VIII度时，本文方法达到收敛所需模 拟次数小于GIRAFFE所需次数; 地震烈度为IX度时, 本文方法达到收玫所需模拟次数等于GIRAFFE所需 次数.

分别应用本文方法与GIRAFFE在地震烈度为VIII 度进行 25 次, IX度时 20次模拟. 收玫指标变化如图11所 示. 可以看出在地震烈度为IX度时, 在 20 次模拟后, 本 文方法与GIRAFFE同时满足Monte Carlo结束条件: $\eta_{\mu}<\xi$ 和 $\eta_{c}<\xi$; 在地震烈度为VIII度时, 在 15 次模拟后, 本文方法计算结果满足结束条件，而GIRAFFE则在 25 次Monte Carlo模拟计算结果才满足结束条件.

图12为地震烈度为VIII, IX度时, 本文方法与GIR$\mathrm{AFFE}$ 各次模拟的管网 $S S I$ 与其均值 $\mu$ 的变化曲线. 从图

表 8 达到收敛条件所需模拟次数

\begin{tabular}{cccc}
\hline 地震烈度 & 本文方法 $\left(n_{1}\right)$ & GIRAFFE $\left(n_{2}\right)$ & 比值 $\left(n_{2} / n_{1}\right)$ \\
\hline VII & 15 & 20 & 1.33 \\
VIII & 15 & 25 & 1.67 \\
IX & 20 & 20 & 1.00 \\
\hline
\end{tabular}

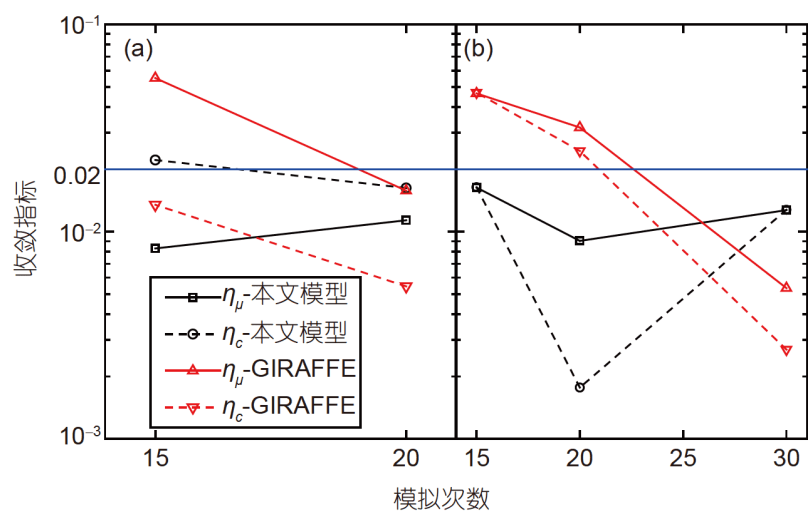

图 11 (网络版彩图)收敛指标 $\eta_{\mu}$ 和 $\eta_{c}$ 变化趋势. (a) 地震烈度 为IX度; (b) 地震烈度为VIII度

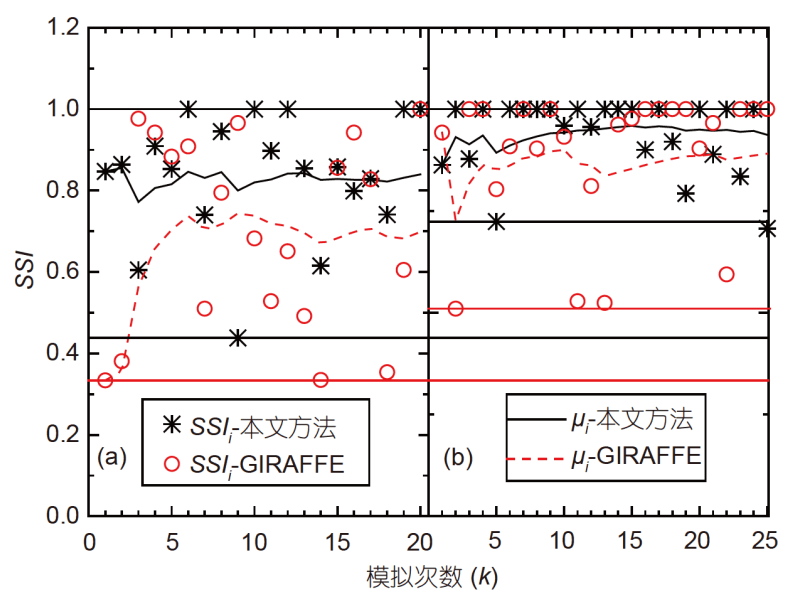

图 12 (网络版彩图)各次模拟 $S S I$ 及均值 $\mu$. (a) 地震烈度为 IX度; (b) 地震烈度为VIII度

12中可以看出, 地震烈度为VIII度时, 本文方法模拟结 果离散性明显比GIRAFFE模拟低；因此，本文方法在 地震烈度为 VIII度时可以以较少的模拟次数达到模拟 结束条件. 地震烈度为IX度时, 本文方法与 GIRAFFE 模拟结果的SSI收玫性相差不大, 但依然比GIRAFFE模 拟结果收敛. 说明本文方法可以通过不多于GIRAFFE 的模拟次数达到模拟结束条件.

\section{4 不同地震烈度下管网供水状态模拟}

本文方法模拟地震烈度为VII，VIII，IX度下供水 管网水力状态. 评估结果如图13所示. 可以看出, 随着 烈度提高, 管道地震害率增大, 节点和管网需水量满足 率SI和SSI随之降低.

如图13所示, 节点11为水源51下游节点, 在地震烈 度为VIII，IX度下需水量满足率不为 1 . 这是由于采用 PDD方法进行水力平差，由于节点11的下游管道破坏， 导致降低了节点11水压, 从而降低配水量. 而节点10则 相反, 节点 10 是因为上游节点 9,13 的节点需水量满足 率降低引起节点 10 的节点需水量满足率下降.

节点 $41,32,35,48,23$ 为节点高程最大的前 5 个节 点, 高程分别为 $30.5,20.5,18.8,18.8,18 \mathrm{~m}$. 如图13(a)所 示, 这 5 个节点在地震烈度为VII度时, 节点需水量满足 率均 $S I$ 小于 1 , 为需水量部分满足节点. 在图6中, 可以 看出, 这 5 个节点的需水量满足率下降较大.

节点 $47,22,9,8,6$ 为节点需水量最大的前 5 个节 点, 需水量分别为 $408,286,276,266,233 \mathrm{~L} / \mathrm{s}$. 如图13所 示, 在地震烈度为VII度时, 节点47的节点需水量满足 

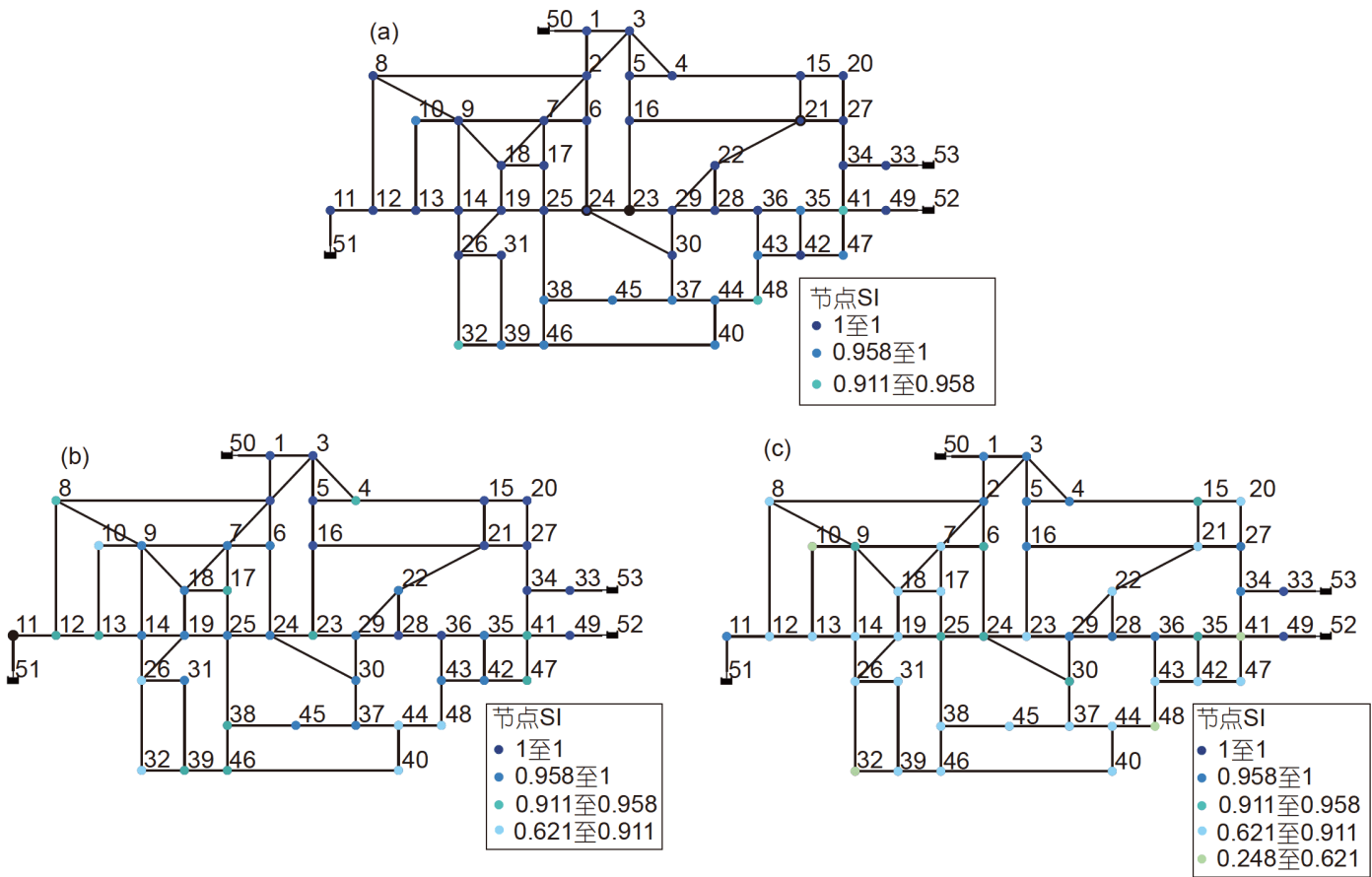

图 13 地震烈度为IX度时供水管网节点满足率分布. (a) 地震烈度为VII度, SSI=0.993; (b) 地震烈度为VIII度, SSI $=0.952$; (c) 地 震烈度为IX度, $S S I=0.837$

率小于 1 ; 地震烈度为VIII, IX度时, 节点 $22,9,8,6$ 需水 量满足率小于 1 .

供水距离同样影响节点需水量满足率均 $S I$, 如节 点 45 高程为 $9.1 \mathrm{~m}$, 需水量为 $38 \mathrm{~L} / \mathrm{s}$. 高程和需水量均不 大, 但其距离水源点较远, 供水距离较长, 因此也容易 在地震后节点需水量满足率均SI降低.

通过以上分析可得到结论: (1) 管道震害率对管网 震后水力状态影响很大, 管道震害率越高, 供水管网节 点满足率越低; (2) 在PDD方法的水力平差中, 即使节点 上游管道未发生破坏, 也有可能因为其他节点的压力影 响而导致实际配水量小于需水量; (3) 节点高程、需水 量以及与水源点距离对节点满足率指标有一定影响, 一 般高程越高, 需水量越大, 与水源点距离越远, 节点满足 率指标 $S$ 越容易降低.

\section{5 总结}

震后管网处于多点漏水, 低压运行状态, 节点需水 量不能完全满足. 本文提出基于压力驱动节点配水量 模型的供水管网震后分析方法，并应用本文提出方法
与美国Cornell大学开发的供水管网震后水力功能分析 软件GIRAFFE软件分别分析某城市供水管网震后供 水能力. 算例分析表明:

（1）本文方法计算得到供水管网震后水力状态与 GIRAFFE软件计算结果的变化规律相同, 当地震烈度 相同时，本文方法与GIRAFFE软件计算得到相同的供 水能力薄弱节点, 节点供水满足率空间分布规律相同;

(2) 基于压力驱动节点配水量模型, 可以同时分析 管道破坏对管道上游和下游节点配水量的影响; 解决 了 GIRAFFE模拟结果中对于震后节点配水量只能 $100 \%$ 或者 0 满足需水量的不足，模拟结果更符合实际 震害情况;

(3) 与GIRAFFE软件相比本文方法计算效率更高, 达到相同模拟收玫条件, GIRAFFE软件所需模拟次数 为本文方法的1.0 1.6倍; 而完成单次模拟所需时间为 本文方法的1.1 2.2倍;

（4）节点高程、需水量以及与水源点距离是影响 供水管网震后对节点的供水能力的主要因素; 节点高 程大、需水量大, 距离水源远的节点是管网震后供水 能力薄弱的节点. 


\section{参考文献}

1 李杰. 生命线工程抗震: 基础理论与应用. 北京: 科学出版社, 2005

2 侯本伟. 城市供水管网抗震能力分析及性能化设计方法研究. 博士学位论文. 北京: 北京工业大学, 2014

3 GRAFFE user's manual Version 4.2, Ithaca: Cornell University, School of Civil \& Environmental Engineering, 2008

4 Shi P X, O'Rourke T D, Wang Y. Simulation of earthquake water supply performance. In: 8th US National Conference on Earthquake Engineering 2006, V 2. Oakland: Earthquake Engineering Research Institute, 2009. 543-552

5 Shi P X, O’Rourke T D. Seismic response modeling of water supply systems. Technical Report MCEER-08-0016. 2008

6 Wang Y, O'Rourke T D. Seismic performance evaluation of water supply systems. Technical Report MCEER-08-0016. 2008

7 Jeon S S, O'Rourke T D. Northridge earthquake effects on pipelines and residential buildings. Bull Seismol Soc Am, 2005, 95: 294-318

8 Wang Y, Au S K. Spatial distribution of water supply reliability and critical links of water supply to crucial water consumers under an earthquake. Reliab Eng Syst Saf, 2009, 94: 534-541

9 马东辉, 韩朝, 王威. 基于GIRAFFE模拟的震后供水管网优化恢复过程研究. 四川建筑科学研究, 2017, 43: 81-85

10 Yoo D G, Kang D S, Kim J H. Seismic reliability assessment model of water supply networks. In: Patterson C L, Struck S D, Murray D J, eds. World Environmental and Water Resources Congress 2013: Showcasing the Future. Reston, Virginia: American Society of Civil Engineers, 2013. 955-966

11 Laucelli D, Giustolisi O. Vulnerability assessment of water distribution networks under seismic actions. J Water Resour Plann Manage, 2014, 141: 04014082

12 Liu J, Yu G P. Iterative methodology of pressure-dependent demand based on EPANET for pressure-deficient water distribution analysis. J Water Resour Plann Manage, 2013, 139: 34-44

13 Liu J, Yu G, Savic D. Deficient-network simulation considering pressure-dependent demand. In: Ma B S, Najafi M, Jiang G S, et al., eds. ICPTT 2011: Sustainable Solutions for Water, Sewer, Gas, and Oil Pipelines. Reston, Virginia: American Society of Civil Engineers, 2011. 886-900

14 Tanyimboh T T, Templeman A B. Seamless pressure-deficient water distribution system model. Proc Inst Civil Eng-Water Manage, 2010, 163: 389-396

15 侯本伟, 杜修力. 地震破坏供水管网低压水力分析. 土木建筑与环境工程, 2013, 35: 36-43

16 Liu M, Giovinazzi S, Lee P. Seismic fragility functions for sewerage pipelines. In: Sever V F, Lynn O, eds. Pipelines 2015: Recent Advances in Underground Pipeline Engineering and Construction. Reston, Virginia: American Society of Civil Engineers, 2015. 291-30

17 Wagner J M, Shamir U, Marks D H. Water distribution reliability: Simulation methods. J Water Resour Plann Manage, 1988, 114: 276-294

18 American Lifelines Alliance. Seismic guidelines for water pipelines. G\&E Report 80.01.01, Revision 0, 2005

19 Federal Emergency Management Agency (FEMA), National Institute of Building Sciences (NIBS). Earthquake Loss Estimation Methodology HAZUS 97: Technical Manual. Washington DC: Federal Emergency Management Agency, 1997

20 侯本伟, 杜修力. 地震破坏管线漏损分析模型对比研究. 地震工程与工程振动, 2013, 33: 67-75

21 Rossman L A. EPANET 2: users' manual version 2.0. United States Environmental Protection Agency, 2000

22 林雨阳. 城市供水管网实时监控系统的设计与监测点的优化布置. 硕士学位论文. 广州: 华南理工大学, 2013 


\title{
Post-earthquake hydraulic analyses of urban water supply network based on pressure drive demand model
}

\author{
HAN Zhao ${ }^{1,2}$, MA DongHui ${ }^{2,3}$, HOU BenWei ${ }^{1} \&$ WANG Wei ${ }^{2,3}$ \\ ${ }^{1}$ College of Architecture and Civil Engineering, Beijing University of Technology, Beijing 100124, China; \\ ${ }^{2}$ Institute of Earthquake Resistance and Disaster Reduction, Beijing University of Technology, Beijing 100124, China; \\ ${ }^{3}$ College of Architecture and Urban Planning, Beijing University of Technology, Beijing 100124, China
}

This study develops a simulation method of water supply network against earthquakes based on pressure drive demand model. For this purpose, Monte Carlo method is used for the probabilistic seismic simulations and the mean system serviceability is obtained as the final analysis result. In each simulation, a post-earthquake water supply network hydraulic model comes into being as a result of random selection of pipelines status, then the system serviceability is calculated through hydraulic analysis based on pressure drive demand model. In case analysis, we use the developed method and GIARFFE software developed by professor O'Rourke from Cornell University to analyze an urban water supply network performance under different earthquake intensities. The results show that: the results between the developed method and GIRAFFE have the same trends, the developed method needs less number of Monte Carlo simulation than GIRAFFE, and less operation time than the GIRAFFE in a single simulation; a damaged pipeline reduces both of upstream and downstream nodes serviceability in the analysis based on pressure drive demand model.

water supply network, post-earthquake hydraulic analysis, Monte Carlo simulation, pressure drive demand doi: 10.1360/N092017-00429 